\title{
Stefan Schmalz
}

\section{An den Grenzen des American Empire Geopolitische Folgen des chinesischen Aufstiegs ${ }^{1}$}

Im Februar 2015 stimmte der argentinische Kongress der Fortführung eines ambitionierten Bauprojekts im strukturschwachen Nordpatagonien zu. Unter Anleitung einiger chinesischer Vorarbeiter hatten argentinische Arbeiter zuvor eine gewaltige Vorrichtung für eine Raumfahrtbodenstation in der Nähe der Provinzhauptstadt Neuquén errichtet. Das Abkommen zum Bau und zur Nutzung der Station mit China hat ein Finanzvolumen von 300 Millionen US-Dollar und eine Laufzeit über 50 Jahre. Die argentinische Regierung verpachtet eine Landfläche von rund 200 Hektar an die chinesische Raumfahrtagentur (CLTC) und garantiert Steuerfreiheit. Im Gegenzug erhält Argentinien ein Nutzungsrecht der Station für 10 Prozent der Gesamtzeit (The Guardian, 26.5.2015). Die Anlage soll bereits 2016 in Betrieb gehen. Das Abkommen ist von großem Interesse. Dieses bezieht sich nicht nur auf eine mögliche militärische Nutzung der Station, die immer wieder von der argentinischen Opposition als Gefahr skizziert wurde. Vielmehr ist die Raumstation Teil eines größeren Abkommens mit der chinesischen Regierung. Aus einer Vielzahl von gemeinsamen Projekten wie etwa chinesischen Investitionen in zwei moderne Wasserkraftwerke sticht hierbei ein Vertrag besonders hervor: Argentinien, das mit einer negativen Leistungsbilanz, einem drastischen Verfall des Peso und einem technischen Staatsbankrott zu kämpfen hatte, unterzeichnete mit China einen Vertrag über einen in Renminbi dotierten Währungsswap über rund 11 Milliarden US-Dollar zwischen den Zentralbanken der beiden Länder. Das Swap-Abkommen versorgte das Land in den Folgemonaten mit dringend benötigter Liquidität und kann zur Finanzierung der umfangreichen Importe aus China genutzt werden. Der Peso-Wechselkurs stabilisierte sich durch den Bailout ,中国制造 (Made in China)“ und ermöglichte der Regierung neue wirtschaftspolitische Handlungsspielräume.

1 Die folgenden Ausführungen stellen einige Ergebnisse meiner Habilitationsschrift dar, die ich im Juli 2014 an der Fakultät für Sozial- und Verhaltenswissenschaften der FriedrichSchiller-Universität Jena eingereicht habe (Schmalz 2014). Die Arbeit wurde durch ein Stipendium des DFG-Kollegs „Postwachstumsgesellschaften” an der Friedrich SchillerUniversität Jena gefördert. 
Die Geschehnisse deuten daraufhin, dass der Aufstieg Chinas in der Weltpolitik und -wirtschaft zu einer Vielzahl von neuen Abhängigkeiten führt, die sich oftmals langsam, ja, manchmal sogar fast vor der globalen Öffentlichkeit verborgen etablieren. Statt vom IWF, bei dem die argentinische Regierung bis vor zehn Jahren tief verschuldet war, kam der Standby-Kredit in einer Krisensituation nun aber direkt von der chinesischen Zentralbank. Und statt eines Strukturanpassungsprogramms wurden milliardenschwere Investitionsprojekte mit chinesischen Unternehmen, darunter auch die Raumfahrtbodenstation, vereinbart. Es ist deshalb von großem Interesse, ob es sich bei solchen Vereinbarungen eher um die Ausnahme oder die Regel im 21. Jahrhundert handeln wird. Im Folgenden wird deshalb untersucht, wie weit Chinas globales Engagement heute fortgeschritten ist und ob es eine Herausforderung für die USA und ihre europäischen Verbündeten darstellt. Um diese Frage zu beantworten, wird zunächst auf einer theoretisch-konzeptionellen Ebene herausgearbeitet, nach welchem Muster der Aufstieg von neuen Weltmächten historisch bisher ablief und wie er sich in der globalen politischen Ökonomie umsetzt. Es werden dann drei Strukturveränderungen diskutiert, die durch Chinas Aufstieg hervorgerufen wurden: Erstens Chinas langsamer Aufstieg zum neuen consumer of last resort der Weltwirtschaft; zweitens Chinas umfangreiche Investitionen in Infrastruktur, Ressourcenvorkommen und Unternehmensanteile im globalen Süden und drittens die neue Rolle der Volksrepublik als Finanzmacht und Kreditgeber. Abschließend werden die Reaktion der USA auf diese Prozesse diskutiert und die Perspektiven des chinesischen Aufstiegs ausgelotet.

\section{Machtverschiebungen in der Weltwirtschaft: Die Dialektik von Geopolitik und Kapitalakkumulation}

Bereits Wladimir Ilijtsch Lenin hatte in seiner Imperialismus-Schrift darauf hingewiesen, dass der Kapitalismus zur kontinuierlichen räumlichen Reorganisation neigt. Eine „gleichmäßige Entwicklung der einzelnen Unternehmungen, Trusts, Industriezweige und Länder“ könne es, so Lenin „unter dem Kapitalismus nicht geben. Vor einem halben Jahrhundert war Deutschland, wenn man seine kapitalistische Macht mit der des damaligen Englands vergleicht, eine klägliche Null; ebenso Japan im Vergleich zu Russland. Ist die Annahme »denkbar«, dass das Kräfteverhältnis zwischen den imperialistischen Mächten nach zehn, zwanzig Jahren unverändert geblieben sein wird? Das ist absolut undenkbar." (Lenin 1917: 75). An der Grunddynamik dieser ungleichen Entwicklung und den daraus resultierenden Verschiebungen von kapitalistischer Macht im Staatensystem hat sich im letzten Jahrhundert wenig geändert. Auch heute ringen staatliche Akteure 
um Einfluss in der Weltwirtschaft und setzen dabei als Grundlage auf eine starke Volkswirtschaft. Lenins Zitat beschreibt letztlich eine Dynamik, die historisch immer wieder in neuer Gestalt auftritt. Großbritannien war bis zu Beginn des 18. Jahrhunderts ein Exporteur von Rohstoffen, die in den Manufakturen in Kontinentaleuropa verarbeitet wurden (List 1841: 126). Erst massive Staatsinterventionen, neue Klassenverhältnisse im Agrarsektor und der Protektionismus förderten die industrielle Entwicklung. Die Dynamik des britischen Industriekapitalismus und der Kolonialismus (insbesondere die Besetzung Indiens) bildeten die Grundlage für das Britische Empire, das zeitweilig ein Drittel des weltweiten Territoriums umfasste. Das britische Freihandelssystem, das auf dem Goldstandard und der balance of power in Europa aufbaute, wurde erst durch nachholende Modernisierungsprozesse in den USA und im Deutschen Reich infrage gestellt (Cox 1987: 151ff.); bis ins frühe 20. Jahrhundert konnte Großbritannien seine zentrale Stellung durch die Vorherrschaft im Finanzsystem und den Ausbau der Flotte aufrechterhalten.

Mit den USA übernahm nach dem Zweiten Weltkrieg ein neuer Staat die Rolle als Hegemonialmacht: Die Vereinigten Staaten waren von einer britischen Kolonie im 19. Jahrhundert zu einem unabhängigen Kontinentalstaat herangewachsen und hatten ihre neue Industrie durch umfangreiche Schutzzölle abgeschirmt (Chang 2002: 24ff.). Auch wenn die Wirtschaftsleistung der USA jene Großbritanniens bereits am Beginn des 20. Jahrhunderts übertraf, konnte das Land erst nach zwei Weltkriegen ein neues institutionelles Regulierungssystem durchsetzen (Bretton Woods, NATO, etc.) und den US-Dollar als Weltwährung etablieren (Arrighi 1994: 269ff.). Dieses baute auf die Verallgemeinerung der fordistischen Konsumgesellschaft und die Integration der früheren Feinde (Japan und Deutschland) in das Militär- und Handelssystem; der Kalte Krieg diente als Klammer, um das Bündnis der westlichen Industrienationen zusammenzuhalten. Derzeit scheint China einen ähnlichen Prozess zu durchlaufen: Das Land befreite sich erst mit der Unabhängigkeit 1949 von der kolonialen Vergangenheit und durchlief seit den späten 1970er Jahren einen raschen kapitalistischen Modernisierungsprozess (Naughton 2006: 85ff.). Die Volksrepublik China ist heute drauf und dran, bei vielen Wirtschaftsindikatoren zu den USA aufzuschließen. Mittlerweile hat der chinesische Staatskapitalismus außerdem begonnen, global zu expandieren; es sind zudem erste institutionelle Regulierungsmuster zu beobachten, die diese Expansionsbewegung strukturieren werden.

Aus den bisherigen Machtverschiebungen im globalen Kapitalismus lassen sich einige Erkenntnisse gewinnen: 1) Der globale Kapitalismus neigte bisher dazu, sich räumlich ungleich zu entwickeln und seinen Schwerpunkt immer wieder zu verlagern. Regelmäßige historische „Dezentrierungen“ der Kapitalakkumulation von einem Zentrum der globalen Ökonomie (z.B. von London) gingen 
dabei stets mit einer Rezentrierung (z.B. nach New York) einher, „so als ob eine Weltwirtschaft nicht ohne Schwerpunkt, ohne Pol existieren könnte“ (Braudel 1986: 78; vgl. auch: Arrighi 1994: 12ff.).

2) Diese globale Dynamik gründete sich darauf, dass es zu einer dynamischen Entwicklung in Randregionen des Kapitalismus kam. Die großen Herausforderer der alten Hegemonialmächte rückten aus der (Semi-)Peripherie der kapitalistischen Weltwirtschaft in ihr Zentrum auf (Chase-Dunn 2005: 179). England war - wie weiter oben notiert - bis zu Beginn des 18. Jahrhunderts ein Rohstofflieferant am Rande des europäischen Handelskapitalismus; die USA (und auch Russland) waren neue Kontinentalstaaten, deren Territorium noch bis Ende des 19. Jahrhundert durch Siedlungsprozesse rasch anwuchs; China (und auch Indien) befreiten sich erst nach dem Zweiten Weltkrieg aus kolonialer Bevormundung. In allen Fällen handelte sich um Prozesse, die durch massive Staatseingriffe vorangetrieben wurden (Chang 2002: 58ff.).

3) Die großen historischen Machtverschiebungen in der Weltwirtschaft und -politik zeichneten sich dabei durch ihre Ungleichzeitigkeit auf unterschiedlichen Ebenen (Militär, Wirtschaft, Finanzen, etc.) aus. Diese Ungleichzeitigkeit beruht auf einer Dialektik von geopolitischer und -ökonomischer Machtlogik: Sobald die räumliche Verlagerung der Kapitalakkumulation in Richtung eines neuen Zentrums fortgeschritten war, untergrub sie die relativ statische geopolitische Ordnung, das globale Institutionengefüge, die militärischen Bündnisstrukturen und sogar die weltweite Finanz- und Währungsarchitektur (Schmalz 2014: 113ff.). In diesem Prozess kam es zu geopolitischen Auseinandersetzungen, in denen Staaten ihre Machtressourcen (Finanzsektor, Militär, etc.) mobilisierten, um ihre Stellung zu verteidigen oder auszubauen. Die Dialektik von geopolitischer und -ökonomischer Machtlogik wurde historisch durch die Entstehung immer komplexerer geopolitischer Ordnungen auf eine neue Stufe gehoben, da hierdurch auch die reibungslose Expansion des globalen Kapitalismus ermöglicht wurde.

4) Die Hegemonialmächte konnten ihre Stellung im Weltsystem nur absichern, wenn sie ein spezifisches Produktions- und Konsumtionsmodell in andere Weltregionen über sehr unterschiedliche Instrumente (Investitionen, Konsummuster, militärische Expansion) diffundierten und systemische Kapazitäten des Weltregierens zur Verfügung stellten (Cox 1987, Arrighi/Silver 1999: 26ff.). Dabei nahmen sie eine Vorbildfunktion für andere Staaten und Gesellschaften an: Wirtschaftlich, weil ihr ökonomisches Entwicklungsmodell imitiert wurde; gesellschaftspolitisch, da sich die Eliten und Mittelschichten meist an den kosmopolitischen Vorbildern in diesen Ländern orientierten; kulturell, da auch ihre Lebensweise (z.B. der american way of life) eine Ausstrahlung entfaltete.

Historisch haben sich die Form und die Logik solcher Umbrüche jedoch immer wieder verändert. Heute ist eine zentrale Frage, ob und wie eine solche 
Verschiebung in einer transnationalisierten Weltwirtschaft möglich ist. Besonders pointiert brachten Leo Panitch und Sam Gindin (2012) eine Überlegung zu diesem Problem aufs Papier: Unter Führung der USA sei spätestens seit dem Ende des Kalten Kriegs ein globales American Empire entstanden, das durch die Macht der US-amerikanischen (Finanz-)Unternehmen, die globale Militärpräsenz und die einzigartigen Kapazitäten des US-Staates zusammengehalten wird. Dies habe auch Einfluss auf die Geopolitik: Interstaatliche Konflikte hätten in der neuen Konstellation abgenommen; die Eliten in den meisten anderen Staaten seien weitgehend in das American Empire integriert (ebd.: 275ff.).

Gegen die These, dass der Kapitalismus heute transnational organisiert ist, ist wenig einzuwenden. Seit 1970 hat sich der Welthandel mehr als versechzigfacht, die Direktinvestitionsströme mehr als verhundertfacht und die Anzahl der transnationalen Unternehmen mehr als verzehnfacht. Ein Drittel des Welthandels wird innerhalb von Konzernen abgewickelt. Auch hat sich der räumliche Maßstab von staatlicher Politik verändert, Institutionen des Weltregierens haben heute eine große Bedeutung (Brand et al. 2007; Zürn 1998). Die Empire-These geht davon aus, dass sich eine transnationale Kapitalistenklasse und Unternehmensnetzwerke herausgebildet haben, die die nationalen Grenzen überwinden. Ihr privilegiertes Verhältnis zu den USA gründe sich in den politischen Unterstützungsleistungen des US-amerikanischen Staates für ihre globale Expansion und der spezifischen Stellung der US-amerikanischen Unternehmen an der Spitze der globalen Wertschöpfungsketten (Panitch/Gindin 2012: 275ff.). Doch die jüngsten politischen Dynamiken deuten daraufhin, dass der US-amerikanische Block von Regierungsapparaten und Konzernen auf Widersacher stößt. Staatenkonflikte zwischen den großen Mächten spielen weiterhin eine gewichtige Rolle. Das American Empire mag zwar die ehemaligen Kontrahenten in Europa und Japan vorerst integriert haben. Doch andere, teils hochgerüstete Staaten wie Russland und China sind zwar wirtschaftlich eng mit den G7-Staaten verknüpft, werden aber dennoch von weitgehend unabhängigen staatlichen Eliten regiert. Zudem hat sich China zu einem Zentrum der globalen Kapitalakkumulation entwickelt, das die Rolle der USA durchaus herausfordern kann. Im Folgenden werden drei zentrale Elemente dieser Transformation skizziert.

\section{Auf dem Weg zum globalen consumer of last resort}

Ein Merkmal der Herausforderung für die Weltmachtrolle der USA ist der Bedeutungsgewinn des chinesischen Binnenmarkts als zentrale Bezugsgröße für ausländische Exporteure und Investoren. Die Rolle der eigenen Volkswirtschaft als Absatzmarkt und Unternehmensstandort war bereits in früheren Hegemonien 
von Bedeutung. Großbritannien setzte zunächst auf ein Freihandelssystem, bei dem es ausländischen Staaten unilateral Zugang zum Binnenmarkt gewährte und im Gegenzug Liberalisierungen forderte (Arrighi 1994: 164ff.). Dieses Vorgehen war aufgrund der Produktivitätsfortschritte der britischen Wirtschaft bis ins späte 19. Jahrhundert äußerst rational und war letztlich nur durch das britische Kolonialreich möglich, das als Absatzmarkt für britische Waren diente und somit die Handelsbilanzdefizite Großbritanniens finanzierte, aber keine ähnlichen Vorzüge wie die ausländischen Staaten erhielt. Die US-Hegemonie baute ebenfalls auf dem Zugang zum Binnenmarkt auf: Der „embedded liberalism“ (Ruggie 1982) unter US-Hegemonie nach dem Zweiten Weltkrieg ermöglichte günstigen Marktzugang für einzelne selektiv ausgewählte Staaten(-gruppen). Hierbei profitierten vor allem die ostasiatischen Länder von den Sonderregelungen dieser Politik des „development by invitation“" (Wallerstein 1979: 80), bei der die Exporterfolge als Grundlage für einen nachholenden Industrialisierungsprozess dienten. Chinas Volkswirtschaft hat sich in die ostasiatische Arbeitsteilung integriert und in den vergangenen Jahren deutlich an Gewicht gewonnen (Hung 2009: 16). Das Land ist heute dabei, auf Kosten der westlichen Industrienationen zum größten Absatzmarkt weltweit aufzusteigen. Bereits 2014 war China mit 13,3 Prozent des globalen BIP die zweitgrößte Volkswirtschaft der Welt. Wenn sich die ökonomischen Turbulenzen der letzten Monate nicht verschärfen, ist es durchaus wahrscheinlich, dass die Volksrepublik sowohl die EU als auch die Vereinigten Staaten irgendwann in den späten 2020er Jahren als größte Wirtschaftsmächte ablösen wird. Das Wachstum des chinesischen Binnenmarkts hat dabei dazu geführt, dass China den USA ihre traditionelle Rolle als consumer of last resort strittig macht. Lange Zeit waren die Vereinigten Staaten - auch aufgrund ihres großen Leistungsbilanzdefizits - der Hauptnachfrager nach Waren aufdem Weltmarkt und konnten diese Rolle politisch instrumentalisieren (Scherrer 2014: 95).

Inzwischen hat das exportorientierte Überschussland China aufgrund seiner hohen Außenhandelsverknüpfungen eine wichtige Rolle als Importeur übernommen. So lagen die chinesischen Importe im Jahr 2014 bereits bei über zwei Dritteln des US-amerikanischen Wertes. In verschiedenen Weltregionen wie Ostasien, Südamerika, Afrika oder Zentralasien ist China deshalb bereits für einige Länder zum wichtigsten Absatzmarkt weltweit aufgestiegen. Dieser Wandel hat welthistorische Bedeutung: Ein solcher Umbruch war das letzte Mal beim Hegemoniewechsel von Großbritannien zu den USA in der ersten Hälfte des 20. Jahrhunderts zu beobachten. Bisher weist das chinesische Wirtschaftsmodell mit Investitionsraten von deutlich über 40 Prozent einen Investitionsüberhang und einen niedrigen Konsumanteil auf. Dies steht im Zusammenhang mit dem Fokus auf massive Investitionen in Infrastruktur, Anlagen und Industriekapazitäten. Deshalb hat China große Mengen an Primärgütern wie Energieressourcen (Erdöl) 
oder Industriemetalle (Eisenerz und Kupfer) von Rohstoffproduzenten in verschiedenen Weltregionen importiert und zu dem Rohstoffboom im vergangenen Jahrzehnt beigetragen (Farooki/Kaplinsky 2012: 66ff.). Außerdem importierte China große Mengen an Maschinen, Fahrzeugen und elektronischen Geräten aus Japan, den USA und Europa. Mittlerweile beginnt China, sich auch zum wichtigen Importeur von Konsumgütern aufzuschwingen. Diese Ausrichtung dürfte in den kommenden Jahren aufgrund des beabsichtigten Umbaus der Wirtschaft hin zu einem konsumgetriebenen Modell noch bedeutender werden.

Noch deutlicher wird die neue Rolle des chinesischen Binnenmarkts jedoch, wenn die globale Wachstumsdynamik betrachtet wird: Bereits jetzt hat das Land die USA als den zentralen Motor der Weltwirtschaft abgelöst. In den Postkrisenjahren 2010 bis 2013 steuerte China durchschnittlich über ein Viertel zum globalen BIP-Wachstum bei, weit mehr als die EU (5,3\%) oder die USA $(13,1 \%)$. Hierauf weist auch die Orientierung großer westlicher Industrieunternehmen hin, die heute große Teile ihrer Profite in China erwirtschaften. Nach Schätzungen des Londoner Analysehauses Evercore ISI machte die Rendite des deutschen Volkswagenkonzerns in der Volksrepublik bis zu 60 Prozent am Jahresprofit aus. Vor dem Abgas-Skandal plante das Unternehmen, zwischen 2015 und 2019 alleine in China 22 Milliarden Euro zu investieren (Die Zeit, 25.12.2014). Solche Zahlen zeichnen einen aktuellen Trend nach: Der Zufluss von Direktinvestitionen in die Volksrepublik lag 2013 mit 117,6 Milliarden US-Dollar bei bereits rund drei Vierteln des Wertes der USA (159 Milliarden US-Dollar). Die Mehrheit dieser Investitionen zielt heute nicht mehr auf das export processing, d.h. auf eine bloße arbeitsintensive Montageproduktion für den Export, sondern richtet sich am dynamischen chinesischen Binnenmarkt aus. Dies hat auch eine klassentheoretische Implikation: Mit den neuen Investitionen entsteht eine neue „innere Bourgeoisie“, die in der chinesischen Volkswirtschaft sehr viel tiefer verankert ist als die „Kompradoren“ der Exportwirtschaft (Poulantzas 1973: 52ff.).

Mit der wachsenden Rolle des chinesischen Marktes zeichnet sich zudem ab, welche Regulierungsfunktion der Staat für den Marktzugang übernehmen wird und wie dies das internationale Handelssystem und die globalen Unternehmensnetzwerke beeinflussen wird. Bisher hat China weder auf eine Politik wie das britische Freihandelssystem noch aufden embedded liberalism US-amerikanischer Art gesetzt: Vielmehr hat der chinesische Staat über ein Dickicht von Regulierungen, Eingriffen und Verflechtungen mit nationalen Unternehmen Einfluss auf das Investitionsverhalten ausländischer Unternehmen ausgeübt und (trotz formaler rechtlicher Gleichstellung) die ungleichen Konkurrenzbedingungen auf dem Binnenmarkt aufrechterhalten. Dies lässt sich z.B. an den Regeln der WTO aufzeigen (Breslin 2007: 100). Von den Liberalisierungen im Dienstleistungssektor nach dem WTO-Beitritt 2002 konnten ausländische Wettbewerber oftmals 
nur bedingt profitieren, da die nationalen Unternehmen über klientelistische Beziehungen zum Staat verfügen. Folglich wird der „politische Kapitalismus“ (Weber 1972: 95ff.) chinesischer Prägung, bei dem die Kapitalakkumulation durch „unübliche Geschäfte mit Regierungsstellen“ organisiert wird, auch für die Struktur des globalen Handelssystems bestimmend sein. Dieses könnte einen fragmentierteren Charakter haben, als die Handelssysteme der Vorgänger: Es ist nicht nur wahrscheinlich, dass der chinesische Staat (wie etwa heute in der Roboterindustrie) den Binnenmarkt nutzen wird, um neue globale Standards zu setzen, sondern er wird - wenn diese Pfadabhängigkeit bestehen bleibt - auch über eine Vielzahl von Einzel- und Sonderabkommen besondere Rechte und Präferenzen an einzelne Staaten und ihre Unternehmen verleihen. Dies könnte letztlich zu einem System konzentrischer Kreise beitragen, bei dem, ähnlich dem imperialen Tributsystem der chinesischen Kaiserdynastien, einige privilegierte Partner wirtschaftlich besonders eng an China angebunden sind.

\section{Die Internationalisierung des chinesischen Staatskapitalismus}

Eine weitere Transformation ist in der Investitionspolitik im Gange. Die chinesischen Konzerne haben sich erst vergleichsweise spät internationalisiert. Erst mit der Go Out-Policy (走出去战 略) im Jahr 1999 gab die Staatsführung den Startschuss für ihr Auslandsengagement. Dieser Prozess wurde staatlich koordiniert und durch Gründung von verschiedenen Institutionen wie dem Staatsfonds China Investment Corporation (2007) vorangetrieben. Die Außenorientierung nahm insbesondere seit der Finanz- und Wirtschaftskrise 2008/09 deutlich zu (Schmalz 2010: 492f.). Dies hat vor allem damit zu tun, dass die chinesische Staats- und Parteiführung lange Zeit sehr vorsichtig in ihrem Auslandsengagement war und keine Zerwürfnisse durch politisch sensible Investitionen riskieren wollte. Nach 2008 öffnete sich jedoch eine Chance für chinesische Unternehmen, da in der Krise auch chinesische Investitionen willkommen geheißen wurden und generell die Leistungsbilanzüberschüsse in stabile Auslandsanlagen investiert werden sollten. Heute ist China je nach Berechnungsart der viert- bzw. sogar zweitgrößte Investitionsgeber weltweit. Die Direktinvestitionen lagen nach offiziellen Quellen im Jahr 2013 mit 90 Milliarden US-Dollar auf einem Rekordniveau, sollen aber nach alternativen Berechnungen der Heritage Foundation (2015) sogar 134,6 Milliarden US-Dollar betragen haben. Insgesamt wurden zwischen 2008 und 2013 rund 430,3 Milliarden US-Dollar (offizielle Quellen) bzw. 675,3 Milliarden US-Dollar (Heritage Foundation) im Ausland investiert.

Insgesamt sind diese Werte jedoch noch deutlich niedriger als jene der USA und der EU, die zudem über umfangreiche bereits bestehende Anlagen im Aus- 
land verfügen: Die chinesischen Bestände an Direktinvestitionen beliefen sich 2013 auf ungefähr ein Zehntel des US-Wertes und ein Drittel des deutschen Bestands. In den globalen Unternehmensnetzwerken behalten amerikanische Unternehmen überdies nach wie vor die Überhand: Auch wenn unter den Top 500-Global Fortune Unternehmen Ende 2014 bereits 98 Konzerne aus China (und 128 aus den USA) waren, halten US-amerikanische Konzerne strategische Anteile von größeren Unternehmen im Ausland und kontrollieren somit wichtige Produktionsnetzwerke (Starrs 2013). Außerdem ist die Mehrzahl der chinesischen Konzerne nach wie vor in den globalen Güterketten auf niedrigen Wertschöpfungsstufen integriert. Die profitabelsten Technologiefirmen und bekanntesten Markenhersteller sind in den USA und anderen Zentrumsstaaten beheimatet (ebd.: 822).

Dennoch haben die chinesischen Investitionen mittlerweile eine kritische Masse erreicht, sodass die etablierten Industrienationen in Europa, Japan und den USA um ihren Einfluss in einigen Weltregionen fürchten. Bei den chinesischen Direktinvestitionsaktivitäten herrscht bisher ein klarer Fokus auf den globalen Süden, Zwischen 2008 und 2013 flossen rund ein Fünftel aller chinesischen Investitionen nach Afrika, beinahe 15 Prozent nach Lateinamerika und fast 13 Prozent nach Ostasien. China hat somit in einigen Weltregionen, z.B. in Afrika sowie in Teilen Südostasiens und des Mittleren Ostens die großen Mächte des Nordens als Hauptinvestitionsgeber abgelöst. Auch wenn der Staat bei der Steuerung dieses Prozesses einen gewissen Kontrollverlust erlitten hat, da etwa mitunter einzelne (halb-)staatliche Unternehmen im Ausland als Konkurrenten auftreten, ist doch das Gros der Investitionen auf(halb)staatliche Unternehmen oder Institutionen zurückzuführen. So sind z.B. die chinesischen Staatsfonds SAFE und CIC für Investitionen in dreistelliger Milliardenhöhe verantwortlich, auch die großen Einkäufe im Ressourcenbereich in den Jahren nach der Krise 2008 erfolgten fast ausschließlich durch Unternehmen wie Sinopec, CNPC oder CNOOC (Heritage Foundation 2015). Die chinesischen Investitionen bestehen zudem vorrangig aus Rohstoff- und Infrastrukturinvestitionen, die zusammen rund zwei Drittel der Gesamtinvestitionen ausmachten.

Sowohl die Rohstoff- als auch die Infrastrukturinvestitionen haben einen geopolitischen Charakter. Dies bedeutet, dass in verschiedenen Ländern des globalen Südens Transportwege, Energieversorgung und Rohstoffzufuhr zusehends von chinesischen Staatsunternehmen kontrolliert werden. Gerade die unzähligen Hafenanlagen, Eisenbahnstrecken, Straßen und Flughäfen, die von chinesischen Investoren weltweit gebaut, modernisiert und oft auch betrieben werden, haben weitgehende geopolitische Folgen. Zunächst hat China in den letzten Jahren verschiedene milliardenschwere Megaprojekte ins Leben gerufen, die die Handelsströme nachhaltig verändern werden. Hierzu zählt zum einen 
das Projekt der Seidenstraße, durch das 65 Länder in Europa und Asien mit Schienen- und Straßennetzen sowie Seehandelsrouten besser verbunden werden sollen (Mayer/Huotari 2015: 37) Ein Vorhaben mit vergleichbarer Tragweite ist ein 40 Mrd. teurer Schiffskanal in Nicaragua. Das Joint Venture zwischen dem nicaraguanischen Staat und der Hongkonger HKND Group soll als Alternative zum Panamakanal dienen (FAZ, 30.6.2015). Ferner hat China im Bereich der Transportinfrastruktur neue Innovationen durchgesetzt. Dies lässt sich z.B. in der Schifffahrt beobachten: Neben modernen Tiefseehäfen betreiben chinesische Reeder auch die technologisch am weitesten fortgeschrittenen Containerschiffe, die oftmals vor Ort gebaut werden. Zuletzt hat die chinesische Regierung mittlerweile Partner gefunden, um gemeinsame Institutionen für derartige grenzüberschreitende Infrastrukturprojekte zu schaffen. Hierunter zählt neben der BRICS-Entwicklungsbank vor allem die Asiatische Infrastrukturbank, der mittlerweile 57 Staaten, darunter sogar europäische Länder wie Deutschland, Frankreich und Italien, beigetreten sind. Mit einem Startkapital von rund 100 Milliarden US-Dollar zielt die in Peking ansässige Institution vor allem auf den Ausbau der Infrastruktur in Asien. Auch wenn sie nicht in direkter Konkurrenz zur Weltbank steht, sind die Finanzmittel - wie bei der BRICS-Entwicklungsbank - jedoch dem Einfluss der USA und Japans entzogen, da beide Staaten keine Mitgliedschaft beabsichtigen.

Die umfangreichen staatsgetriebenen Investitionen sind sicherlich nur der Anfang einer umfassenden Strategie der Internationalisierung. Ähnlich wie bei früheren Hegemoniekonstellationen wird über Direktinvestitionen die eigene Machtposition gestärkt, wobei die Investitionsflüsse eine spezifische Struktur des Weltmarkts abstützten. Im Britischen Empire dienten viele solcher überwiegend privaten Direkt- und Portfolioinvestitionen sowie Kredite zur Exploration und zum Abtransport von Rohstoffen - und dies gerade auch in Einflusszonen außerhalb der kolonialen Besitzungen wie in Lateinamerika (Donghi 1991: 256f.). Die britischen Investitionen sollten zudem die eigene Vorherrschaft in der Konkurrenz mit den aufstrebenden imperialistischen Mächten im späten 19. Jahrhundert verankern; zeugten jedoch von einem asymmetrischen Machtverhältnis, durch das den Peripherieländern wenig Entwicklungsmöglichkeiten geboten wurden. Ähnlich wurde die US-Hegemonie nach dem Zweiten Weltkrieg durch einen Schub von Investitionen transnationaler Konzerne gefestigt. Dabei ging es vor allem um den Zugang zu nationalen Märkten und der Kontrolle von Unternehmensnetzwerken, wie etwa bei den umfangreichen Investitionen von US-Unternehmen im fordistischen Nachkriegseuropa (Poulantzas 1973). Das American Empire erhielt in dieser Phase seine wirtschaftliche Grundlage. China hingegen setzt bisher primär auf staatliche Investitionen im Ressourcensektor und im Infrastrukturbereich; dabei wird auf multilaterale Institutionen und Koope- 
rationsprojekte mit oftmals (semi-)peripheren Staaten abgezielt. Der chinesische Staatskapitalismus expandiert also im transnationalen Raum, indem öffentliche Güter (insbesondere Transportinfrastruktur) zur Verfügung gestellt werden. Anders als in der Phase des klassischen Imperialismus können diese Infrastrukturprojekte jedoch durchaus für eine nachholende Modernisierungsstrategie genutzt werden, wie etwa das Beispiel Ecuadors verdeutlicht (New York Times, 26.7.2015). Zugleich werden jedoch elementare chinesische Interessen bedient, so dass Eigen- und Allgemeininteressen verbunden werden. Dass diese „statist globalization“" (Harris 2009) zukünftig verstärkt durch private Investitionen von Konglomeraten in höheren Wertschöpfungsstufen (und in den Zentrumsstaaten) wie Alibaba Group oder Lenovo ergänzt wird, ist bereits absehbar; bisher haben die chinesischen Investitionen aber zu einer relativ erfolgreichen, kooperativen Zusammenarbeit mit anderen Staaten des globalen Südens beigetragen.

\section{Auf dem Weg zur Finanzmacht}

Die gewaltigen Exportüberschüsse der chinesischen Volkswirtschaft haben dazu geführt, dass China Währungsreserven von derzeit 3,6 Billionen US-Dollar (Sep. 2015) angehäuft hat. China ist deshalb ein Gläubigerstaat: Derzeit schuldet der US-amerikanische Staat chinesischen, überwiegend (halb)staatlichen Gläubigern rund 1,3 Billionen US-Dollar, die EU-Staaten kamen nach Schätzungen aus dem Jahr 2013 zusammen auf über 550 Milliarden US-Dollar (Meunier et al. 2014: 112). Seit 2008/09 versucht die chinesische Regierung, diese Anlagen (u.a. in Direktinvestitionen) zu diversifizieren und den Renminbi zu einer Weltwährung aufzubauen (Eichengreen/Kawai 2014).

Ein Weg der Diversifizierung besteht dabei in der Kreditvergabe - insbesondere an Schuldner in der „Dritten Welt“ (Gallagher et al. 2012, Wolf et al. 2013). Die Zahlen über die Kreditvergabe sind intransparent und umstritten, außerdem gehen aufgrund der komplexen Deals manchmal Kredite, Entwicklungshilfe und Investitionen ineinander über. Nach einer Studie der Rand Corporation aus dem Jahr 2014 hatten chinesische Gläubiger alleine 2010 und 2011 Zusagen über rund 357,9 Milliarden US-Dollar gemacht, was das Kreditvolumen der Weltbank und des IWF in dieser Zeit übertraf. Auch wenn die bisher geleisteten Zahlungen nach Angaben der Rand-Corporation nur bei rund einem Zehntel lagen, da viele dieser Projekte erst jetzt oder in einigen Jahren anlaufen, ist die chinesische Finanzmacht in einigen Weltregionen besonders erdrückend. In Lateinamerika hatten chinesische Staatsinstitutionen einer Studie zufolge zwischen 2005 und 2011 rund 75,3 Milliarden US-Dollar Kredite vergeben und damit mehr als die Weltbank oder die Interamerican Development Bank (Gallagher 
et al. 2012: 17). Die Kreditvergabe folgte dabei auch strategischen Erwägungen. Unter den Empfängern von chinesischen Krediten und Entwicklungshilfezahlungen befanden sich vor allem Erdöl- bzw. Rohstoffexporteure und potenzielle geopolitische Verbündete. Die Top Ten umfasste Staaten wie Venezuela (106 Milliarden US-Dollar), Iran (89 Milliarden US-Dollar), Nigeria (72 Milliarden US-Dollar), Pakistan (66 Milliarden US-Dollar), Brasilien (40 Milliarden USDollar) und Indonesien (38 Milliarden US-Dollar) (Wolf et al. 2013: 50). Ähnlich wie bei den Direktinvestitionen sind die meisten der Kreditzusagen an konkrete Infrastruktur- oder Ressourcenabbauprojekte gebunden. Letztlich ersetzt China also in einigen Weltregionen die traditionellen Entwicklungshilfeagenturen und Kreditinstitutionen. Dies drückt sich auch in der Restrukturierung der IWF-Kreditvergabe aus: Heute gehen über drei Viertel der Mittel nicht mehr in den globalen Süden, sondern in die EU und ihre Anrainerstaaten (IMF 2015). Die Konditionen der chinesischen Kredite werden von vielen Schuldnern als günstiger wahrgenommen.

Durch die Strategie zur Internationalisierung des Renminbi ist ein Wandel in den internationalen Finanzbeziehungen eingetreten (Liao/McDowell 2015; Schmalz 2014: 431ff.). China stellt seinen Außenhandel langsam auf RenminbiHandel um. Die chinesische Wirtschaft wickelte 2014 bereits mehr als ein Fünftel ihres Außenhandels in Renminbi ab. Zur Etablierung des Renminbi als Handelswährung haben auch Swap-Abkommen mit 30 Staaten und der Eurozone im Wert über beinahe 3,1 Billionen Renminbi beigetragen. Hierdurch ist der Renminbi bereits Ende 2013 mit einem Anteil von 8,7 Prozent - zwar weit hinter dem Dollar (81,1\%), aber schon vor dem Euro - zur zweitwichtigsten Handelswährung aufgestiegen. Ferner hat die chinesische Staatsführung auch die Entstehung der Renminbi-Anleihe-, Spar- und Währungsmärkte vorangetrieben. In den größten Offshore-Renminbi-Märkten wie Hongkong und London, dem von der chinesischen Parteiführung favorisierten ausländischen Renminbi-Offshore-Markt, wurden im September 2015 bereits Kontrakte im Wert von 192,5 Milliarden US-Dollar gehandelt. Der Shanghaier Finanzplatz erhielt einen weiteren Schub durch den Beschluss, in der Metropole eine Pilot-Freihandelszone zu etablieren. Der Yuan ist heute bereits zur fünftwichtigsten Transaktionswährung aufgestiegen, im Zahlungsverkehr zwischen China und den Anrainerstaaten in Ostasien rangiert der Renminbi bereits auf Platz 1 (Financial Times, 27.5.2015), sodass es wahrscheinlich ist, dass sich in Ostasien in den nächsten Jahren ein RenminbiBlock herausbilden wird.

Allerdings ist die Währung weiterhin nicht konvertibel. Kapitalverkehrskontrollen schirmen den Zugang für ausländische Anleger zu den Finanzmärkten in Festlandchina - mit Ausnahme des Shanghaier Aktien- und Anleihemarkts - weitgehend ab. Die Börsenkrise im Sommer 2015 wird eine allzu rasche Li- 
beralisierung der chinesischen Finanzmärkte zusätzlich abbremsen. Folglich ist anzunehmen, dass der Renminbi zunächst seine Position als Handels- und Transaktionswährung ausbauen, aber nur langsam zu einer wichtigen globalen Anlagewährung werden wird. Auch wenn die Bedeutung der chinesischen Währung für den Handel und die Finanzwelt bisher in atemberaubender Geschwindigkeit anwuchs, bedeutet dies dennoch, dass der US-amerikanische Finanzmarkt zunächst nicht durch die Finanzplätze in Hongkong, Shanghai oder Shenzhen herausgefordert wird. Das Eingangsbeispiel zu Argentinien zeigt jedoch auch, dass die staatsgetriebene Internationalisierungsstrategie erste Auswirkungen hinterlässt, da die Renminbi-Swap-Abkommen mittlerweile nicht nur zur Handelsfinanzierung genutzt werden, sondern auch in Krisensituationen als Stützungsmaßnahme wirken können.

Die Tragweite der Internationalisierung des Renminbi sollte nicht unterschätzt werden: Es ist ein Allgemeinplatz geworden, dass sich ein großer Teil der strukturellen Macht der Vereinigten Staaten auf die exzeptionelle Rolle des US-Dollars und des amerikanischen Finanzplatzes gründet (Gowan 1999: 19ff.; Strange 1988: 107ff.). Zwar ist durchaus umstritten, ob die Finanzialisierung der US-Ökonomie letztlich nicht bereits ein Anzeichen des Niedergangs der US-amerikanischen Hegemonie ist und deshalb als ein äußerst fragiler, wenig nachhaltiger Weg zur Restauration der Sonderstellung der USA im kapitalistischen Weltsystem seit den 1980er Jahren wahrgenommen werden sollte (Arrighi 1994: 314ff.). Aber die Vorteile für den US-amerikanischen Staat wie günstige Konditionen der Schuldenaufnahme, Seigniorage-Einnahmen des US-Dollars oder die Macht der Fed, die globale Zinsentwicklung zu steuern, sind gewaltig (Ivanova 2013). Die Vorherrschaft im Finanzsektor war auch ein Anzeichen der britischen Hegemonie. Durch den Goldstandard und den Londoner Finanzplatz stand Großbritannien im Zentrum der internationalen Finanz- und Währungsarchitektur. Auch hier war der Finanzsektor erst seit den 1870er Jahren voll entfaltet, sodass die britischen Dienstleistungen durch „Schifffahrt, Handel und Vermittlung im Weltzahlungssystem (...) unentbehrlich (wurden). Wenn London wirklich jemals der Mittelpunkt der Weltwirtschaft gewesen ist, dann zwischen 1870 und 1913." (Hobsbawm 1969: 154)

Wie die chinesische Finanzmacht sich global entfalten wird, hängt letztlich von den weiteren Liberalisierungsschritten der chinesischen Staatsführung ab. Bisher scheint sich ein komplexes System von bilateralen Kreditbeziehungen, Swap-Abkommen und regionalen Finanzinstitutionen wie der BRICS-Entwicklungsbank herauszubilden. Dieses System wirkt letztlich als eine Alternative zu den traditionellen internationalen Finanzinstitutionen wie Weltbank und IWF, die sich als weitgehend reformunfähig erwiesen haben. Auch wenn der chinesischen Regierung eigentlich ein globales Finanzsystem mit einem reformierten 
IWF mit SZR als Weltwährung vorschwebt - ähnlich den Vorschlägen von John Maynard Keynes zur Schaffung des Bancor auf dem Bretton-Woods-Gipfel 1944 -, könnten die milliardenschweren Gold- und Rohstoffkäufe durch die chinesische Zentralbank und andere staatliche Institutionen perspektivisch eine Art ostasiatisches Bretton-Woods-System etablieren, in dem ein konvertibler Renminbi als Ankerwährung an einen Rohstoffkorb gekoppelt wird und Krisenstaaten über Swap-Abkommen mit Liquidität versorgt werden.

\section{Zwischen Wirtschaftskrise und Kaltem Krieg}

Die Volksrepublik China ist dabei, ihre wachsende wirtschaftliche Bedeutung nun auch in strukturelle Macht im globalen Kapitalismus umzusetzen. Neben der Anziehungskraft, die der wachsende Binnenmarkt auf ausländische Exporteure und Investoren ausübt, existieren mittlerweile Ansätze in der Außenwirtschaftspolitik des Landes (z.B. die gezielte Infrastrukturpolitik und Kreditvergabe), die handfeste geopolitische Auswirkungen haben werden. Die wirtschaftliche Vorherrschaft der USA erodiert dabei an den Rändern des American Empire; z.B. wenn sich in Ostasien ein neuer Renminbi-Block herausbildet, in Zentralasien gewaltige Infrastrukturprojekte entstehen, die von Peking finanziert werden, einzelne südamerikanische Länder sich massiv bei chinesischen Gläubigern (Ecuador, Venezuela, etc.) verschulden, und für verschiedene Länder in Subsahara-Afrika (z.B. Angola) China schon lange der Hauptexportmarkt ist. Die staatsgetriebene Globalisierung des „Sinocapitalism“ (McNally 2012) vollzieht sich dabei vor allem im Globalen Süden.

Doch dieser Prozess ist noch im Anfangsstadium und bei weitem kein Selbstläufer. Es gibt interne Hindernisse und externe Interventionen, die den chinesischen Machtzuwachs abbremsen könnten. Zunächst weist das chinesische Wirtschaftsmodell deutliche Strukturprobleme auf (Pettis 2013). Der Umbau des investitionsgetriebenen und exportorientierten Wirtschaftsmodells zu einem stärker konsum- und binnenmarktorientierten Modell ist Blockaden ausgesetzt: Es sind große Überkapazitäten in unterschiedlichen Wirtschaftssektoren (Investitionsgüter, Kapitalmärkte, Immobilienmarkt, etc.) entstanden; die Konsumquote ist bisher nur geringfügig gestiegen. Die Wachstumsraten in verschiedenen Regionen des Landes (Nordosten, Süden, etc.) sind deutlich gefallen oder stagnieren sogar; die Wirtschaftsentwicklung wird derzeit vor allem von den Binnenprovinzen getragen. Der Börsencrash im Sommer des Jahres weist zudem auf die Gefahren überhitzter Märkte und spekulativer Überhänge hin. Dass die Parteiführung ihre jährliches Wachstumsziel für den XIII. Fünfjahresplan (2016-2020) auf 6,5 Prozent des BIP herabsetzt, ist als Eingeständnis zu werten, 
dass das Hochwachstum nicht mehr fortsetzbar ist und sich dauerhaft abschwächen wird. China wird die USA und die EU in den späten 2020er Jahren als größte Volkswirtschaften ablösen, aber nicht in ähnlich hoher Geschwindigkeit die Strukturen der Weltwirtschaft verändern können. Zusätzlich hierzu fehlen China - von der zentralasiatischen Shanghai Cooperation Organization unter Einschluss Russlands abgesehen - vergleichbare Bündnissysteme wie jene der USA, um eine neue Hegemonie durchsetzen zu können.

Außerdem haben die Vereinigten Staaten ihre Machtbasis reorganisiert, um der chinesischen Herausforderung zu begegnen. Dabei handelte es sich um eine mehrdimensionale Strategie, die von systematischer Exportförderung (National Export Initiative) sowie Freihandels- und Investitionsabkommen (TTIP, Trans-Pacific Partnership und TISA) zur Stabilisierung der ökonomischen Basis des American Empire über die Revitalisierung des Finanzsektors durch gezielte Rettungsmaßnahmen und lockere Geldpolitik bis hin zur Schiefergasförderung reicht, um die Abhängigkeit von teuren Energieimporten zu verringern. Die wichtigste Komponente bestand indes in einer militärischen Neuausrichtung. Spätestens seit 2010 leitete die US-amerikanische Regierung in der Außenpolitik eine strategische Aufwertung der pazifischen Region, insbesondere Ostasiens, ein (Clinton 2011). Ostasien wurde nun zum pivot, zum Angelpunkt, der US-amerikanischen Strategie erhoben. Die U.S. Army erweitert seitdem ihre militärischen Kapazitäten in der ostasiatischen Region (Marines in Australien, Kampfschiffe in Singapur, neue Militärbasen in den Philippinen, etc.).

Außerdem unterstützten die USA ihre ostasiatischen Verbündeten Japan, Vietnam und auch die Philippinen, in den Territorialstreitigkeiten mit China. Die US-Politik gleicht hierbei der klassischen Eindämmungspolitik aus dem Kalten Krieg (Friedberg 2011). So führten die USA mehrere gemeinsame Manöver mit Südkorea oder Vietnam durch. Japans Politik, das Verbot von Auslandsinterventionen aus der Verfassung zu streichen, wurde von den USA gefördert. In den Konflikten wirkten die Vereinigten Staaten auch nicht mäßigend, sondern unterstützten z.B. nach der Errichtung der chinesischen „Flugverbotszone“ im chinesischen Meer Japan im Konflikt mit der Volksrepublik. Die Konfliktlinien äußern sich zudem in einem Rüstungswettlauf, bei dem China und auch andere Länder wie Vietnam, Südkorea und Indonesien ihren Rüstungsetat regelmäßig aufstocken. Die chinesische Regierung begann vor dem Hintergrund der sich zuspitzenden Territorialkonflikte, ihre Militärstrategie ebenfalls neu auszurichten. Die Staats- und Parteiführung versucht nun, China (das erste Mal seit rund 600 Jahren) als Seemacht zu etablieren, die territorialen Verluste seit Ende des 19. Jahrhunderts rückgängig zu machen und Zugang zu den Ressourcen in der Region zu erhalten. Neben aktiven territorialen Grenzziehungen wie der Aufschüttung von künstlichen Inseln im südchinesischen Meer und eher sym- 
bolischen Manövern, etwa der Inbetriebnahme des Flugzeugträgers Liaoning im Jahr 2012, wurde unter der Staats- und Parteiführung unter Xi Jinping und Li Keqiang die größte Reform der Volksarmee seit Jahrzehnten eingeleitet, mit der die Kommandostrukturen von Flotte und Luftwaffe effizienter gestaltet und die notwendigen Kapazitäten für Auslandseinsätze aufgebaut werden sollen.

Die neue Konstellation in Ostasien scheint zu einem dauerhaften Zustand zu werden. Es ist bisher von keiner Seite ein Einlenken in den Territorialkonflikten oder gar eine Abrüstung zu beobachten. Folglich könnte Ostasien der Schauplatz eines neuen „capitalist cold war“ (D’Aveni 2012) werden, in dem China perspektivisch versuchen wird, seinen wachsenden wirtschaftlichen Einfluss geltend zu machen, um den Ring von US-amerikanischen Verbündeten aufzubrechen. Der Schulterschluss zwischen Russland und China, der sich in gemeinsamen militärischen Manövern, großen Infrastrukturprojekten wie der Modernisierung der transsibirischen Eisenbahn und milliardenschweren Erdgasdeals äußert, führt in Kontinentalasien zu einer neuen geopolitischen Situation. Bisher stehen zumindest in Ostasien die Zeichen auf Konfrontation ohne »heiße« kriegerische Konflikte; ob und wann Bewegung in die starren Lager kommen wird, ist noch nicht absehbar. Es ist eine Rechnung mit vielen Unbekannten. Wird der Ring US-amerikanischer Verbündeter in Ostasien halten? Was ist, wenn sich die chinesischen Investitionen in Südamerika oder Subsahara-Afrika in neuen Allianzen niederschlagen? Auch wenn wenig dafür spricht, dass China die USA in ihrer hegemonialen Rolle kurz- oder mittelfristig ablösen wird, spricht noch weniger dafür, dass die USA diese Rolle ungebrochen behalten werden. Das American Empire ist an seine Grenzen geraten; die vermeintliche Gewisheit, dass der transnationale Kapitalismus keine territorialen Konflikte kennt, wurde durch die Geschichte als ein Irrglaube entlarvt.

\section{Literatur}

Arrighi, Giovanni (1994): The Long Twentieth Century. Money, Power, and the Origins of Our Times. London.

-/Silver, Beverly J. (1999): Introduction. In: dies. (Hg.): Chaos and Governance in the Modern World System. Minneapolis: 1-36.

Brand, Ulrich/Görg, Christoph/Wissen, Markus (2007): Verdichtungen zweiter Ordnung. Die Internationalisierung des Staates aus einer neo-poulantzianischen Perspektive. In: Prokla 37(2): 217-234.

Braudel, Fernand (1986): Die Dynamik des Kapitalismus. Stuttgart.

Breslin, Shaun (2007): China and the Global Political Economy. Basingstoke-New York.

Chang, Ha-Joon (2007): Kicking Away the Ladder. Development Strategy in Historical Perspective. London.

Chase-Dunn, Christopher (2005): Social Evolution and the Future of World Society. In: Journal of World-Systems Research, 9(2): 171-192. 
Clinton, Hilary (2011): America's Pacific Century. In: Foreign Policy. October 11, 2011. URL: http://www.foreignpolicy.com/articles/2011/10/11/americas_pacific_century, Zugriff: 3.11.2015.

Cox, Robert W. (1987): Production, Power, and World Order. Social Forces in the Making of History. New York.

D'Aveni, Richard A. (2012): Strategic Capitalism. The New Economic Strategy for Winning the Capitalist Cold War. New York.

Donghi, Tulio Halperin (1991): Geschichte Lateinamerikas von der Unabhängigkeit bis zur Gegenwart. Frankfurt/M.

Eichengreen, Barry J./Kawai, Masahiro (2014): Issues for Renminbi Internationalization: An Overview. ADBI Working Paper No. 454. URL: http://www.adbi.org/workingpaper/2014/01/20/6112.issues.renminbi.internationalization.overview/, Zugriff: 3.11.2015.

Farooki, Masuma/Kaplinsky, Raphael (2012): The Impact of China on Global Commodity Prices. The Global Reshaping of the Resource Sector. London.

Friedberg, Aaron L. (2011): Bucking Beijing. An Alternative U.S. China Policy. In: Foreign Policy 91(5): 48-58.

Gallagher, Kevin P./Irwin, Amos/Koleski, Katherine (2012): The New Banks in Town: Chinese Finance in Latin America. China and Latin America Report. URL: http://www.thedialogue. org/PublicationFiles/TheNewBanksinTown-FullTextnewversion.pdf, Zugriff: 3.11.2015.

Gowan, Peter. (1999): The Global Gamble. Washington's Faustian Bid for World Dominance. London-New York.

Harris, Jerry (2009): Statist Globalization in China, Russia and the Gulf States. In: Science and Society, 73(1): 6-33.

Heritage Foundation (2015): China Global Investment Tracker. URL: http://www.heritage.org/ research/projects/china-global-investment-tracker-interactive-map, Zugriff: 3.11.2015.

Hobsbawm, Eric. (1969): Industrie und Empire. Britische Wirtschaftsgeschichte seit 1750. Frankfurt/M.

Hung, Ho-fung (2009): America's Head Servant. The PRC's Dilemma in the Global Crisis. In: New Left Review, 50(6): 5-25.

IMF (2015): Total IMF Credit Outstanding for all members from 1984-2015. URL: http://www. imf.org/external/np/fin/tad/extcred1.aspx, Zugriff: 3.11.2015.

Ivanova, Maria (2013): The Dollar as World Money. In: Science \& Society, 77(1): 44-71.

Lenin, W. I. (1917): Der Imperialismus als höchstes Stadium des Kapitalismus. Werke Bd. 22. Berlin 1960: 191-309.

Liao, Steven/McDowell, Daniel (2015): Redback Rising: China's Bilateral Swap Agreements and RMB Internationalization. In: International Studies Quarterly, 59(3): 401-422.

List, Friedrich (1841): Das nationale System der politischen Ökonomie. In: Wendler, E. (Hg.): Friedrich List: Das nationale System der politischen Ökonomie. Baden-Baden 2008: 53-394.

Mayer, Maximilian/Huotari, Mikko (2015): China: Geopolitik durch Infrastruktur. In: Blätter für Deutsche und Internationale Politik, 60(7): 37-40.

McNally, Christopher A. (2012): Sino-Capitalism: China’s Reemergence and the International Political Economy. In: World Politics, 64(4): 741-776.

Meunier, Sophie/Burgoon, Brian/Jacoby, Wade (2014): The politics of hosting Chinese investment in Europe-an introduction. In: Asia Europe Journal, 12(1-2), 109-126.

Naughton, Barry (2006): The Chinese economy. Transitions to growth. Cambridge: MIT Press. Panitch, Leo/Gindin, Sam (2012): The making of global capitalism. The political economy of American empire. London-Brooklyn.

Pettis, Michael (2013): Avoiding the fall. China's economic restructuring. Washington/DC. 
Poulantzas, Nicos (1973): Die Internationalisierung der kapitalistischen Verhältnisse und der Nationalstaat. In: -/Hirsch, Joachim/Jessop, Bob (2001): Die Zukunft des Staates. Denationalisierung, Internationalisierung, Renationalisierung, Hamburg: 19-69.

Ruggie, John Gerard (1982): International Regimes, Transactions, and Change: Embedded Liberalism in the Postwar Economic Order. In: International Organization, 36(2): 379-415.

Scherrer, Christoph (2014): Die Post-hegemoniale USA? In: Bieling, Hans-Jürgen/Haas, Tobias/ Lux, Julia (Hg.): Die Internationale Politische Ökonomie nach der Weltfinanzkrise. Sonderheft der Zeitschrift für Außen-und Sicherheitspolitik. Wiesbaden: 89-107.

Schmalz, Stefan (2010): Chinas neue Rolle im globalen Kapitalismus. In: Prokla 40(4): 483-503. - (2014): Machtverschiebungen im Weltsystem. Der Aufstieg Chinas im Kontext der globalen Finanzund Wirtschaftskrise seit 2008, bisher unveröffentlichte Habilitationsschrift an der Fakultät für Sozial- und Verhaltenswissenschaften der Friedrich Schiller-Universität Jena.

Starrs, Sean (2013): American Economic Power Hasn't Declined - It Globalized! Summoning the Data and Taking Globalization Seriously. In: International Studies 2uarterly 57(4): 817-830.

Strange, Susan. (1988): States and Markets. London-New York.

Wallerstein, Immanuel (1979): The capitalist world-economy. Essays. Cambridge/MA.

Weber, Max (1972): Wirtschaft und Gesellschaft. Grundriss einer verstehende Soziologie. 5., rev. Auflage, Tübingen: Mohr Siebeck.

Wolf, Charles/Wang, Xiao/Warner, Eric (2013): China's foreign aid and government-sponsored investment activities. Scale, content, destinations, and implications. Rand Corporation research report series No. 118. URL: http://www.rand.org/pubs/research_reports/RR118.html, Zugriff: 3.11 .2015 .

Zürn, Michael (1998): Regieren jenseits des Nationalstaates. Globalisierung und Denationalisierung als Chance. Frankfurt/M.

\section{Verlag Westfälisches DampfBoot}

\section{Tobias ten Brink}

\section{Geopolitik}

Geschichte und Gegenwart

kapitalistischer Staatenkonkurrenz

Mit einem Vorwort von Bob Jessop

(Theorie und Geschichte

der bürgerlichen Gesellschaft Band 23)

2008 - 307 Seiten - 27,90€

ISBN: 978-3-89691-123-0

„ten Brink erneuert auf erfolgreiche Art und Weise den Begriff des Imperialismus als ein wissenschaftliches Konzept und entwickelt einen spezifischen, geschichtlich informierten, analytischen Ansatz, der auf einer detaillierten Kenntnis der umfangreichen theoretischhistorischen Literatur und neuerer empirischer Forschungsergebnisse beruht".

Bob Jessop, Prof. Lancaster University

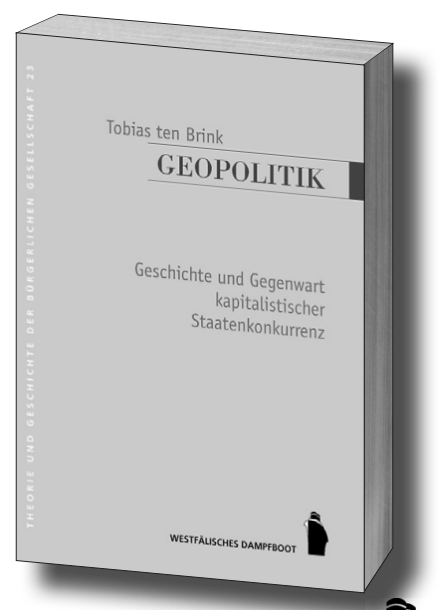

2 